\title{
Re-thinking local causality
}

\author{
Simon Friederich \\ email@simonfriederich.eu \\ University of Groningen, University College Groningen, Hoendiepskade \\ 23/24, NL-9718 BG Groningen and Faculty of Philosophy, Oude \\ Boteringestraat 52, NL-9712 GL Groningen, The Netherlands ${ }^{1}$
}

\begin{abstract}
:
There is widespread belief in a tension between quantum theory and special relativity, motivated by the idea that quantum theory violates J. S. Bell's criterion of local causality, which is meant to implement the causal structure of relativistic space-time. This paper argues that if one takes the essential intuitive idea behind local causality to be that probabilities in a locally causal theory depend only on what occurs in the backward light cone and if one regards objective probability as what imposes constraints on rational credence along the lines of David Lewis' Principal Principle, then one arrives at the view that whether or not Bell's criterion holds is irrelevant for whether or not local causality holds. The assumptions on which this argument rests are highlighted, and those that may seem controversial are motivated.
\end{abstract}

\section{Introduction}

Most physicists agree that quantum theory and special relativity are successfully combined in the theories of modern elementary particle physics and, a fortiori, perfectly compatible. However, the view that there exists a profound tension between the two does have its supporters, among them some of the most eminent researchers in the field of quantum foundations such as, perhaps most famously, John S. Bell. According to Bell, there is "an apparent incompatibility, at the deepest level, between the two fundamental pillars of contemporary theory" ([Bell 2004] p. 172), where by the "two fundamental pillars" he means quantum theory and relativity theory.

Roughly speaking, there are two main reasons found in the literature to be worried about the compatibility between quantum theory and special relativity. The first is that orthodox quantum theory features the notorious collapse of the wave function as an essential ingredient. Collapse amounts to an instantaneous, discontinuous change of the state assigned to a quantum system. The state is subjected to collapse whenever the system is measured, i.e. new information as regards the values of its dynamical variables is obtained. The worry arising from collapse is that it seems to require a

\footnotetext{
${ }^{1}$ work carried out at the University of Göttingen
} 
preferred reference frame and therefore an absolute simultaneity relation. This requirement clashes with the principles of special relativity, according to which all (inertial) frames are on the same conceptual footing and simultaneity among events is frame-relative.

Bell himself, however, does not regard collapse and its instantaneous character as giving rise to an unavoidable incompatibility between quantum theory and special relativity. The suggested difficulty can be avoided if, in his own words, "we do not grant beable status to the wave function" ([Bell 2004] p. 53, for more on Bell's notion "beable" see below), that is, if we deny that quantum states ("wave functions" in particular) correspond to objective properties of the systems they are assigned to. Since there are excellent independent reasons "not [to] grant beable status to the wave function" (though this move admittedly faces its challenges ${ }^{2}$ ), I feel free to ignore this reason for doubting the compatibility of quantum theory and special relativity in the present paper.

The second and, according to many writers, much more profound reason for believing that quantum theory and special relativity are in conflict arises from the fact that quantum theory postulates correlations which violate a criterion proposed by J.S. Bell he refers to as local causality. This criterion is a formally precise implementation of the intuitive idea that in a theory that is supposed to be compatible with the space-time structure of special relativity, in Bell's words "[t]he direct causes (and effects) of events are near by, and even the indirect causes (and effects) are no further away than permitted by the velocity of light." ([Bell 2004] p. 239) Bell's own famous theorem ${ }^{3}$ states that any theory which respects this probabilistic implementation cannot possibly reproduce the quantum correlations. Thus, according to this theorem, neither quantum theory itself nor any (possibly more fundamental future) theory which reproduces the predictions of quantum theory can be locally causal in the sense of that criterion. Since the quantum theoretical predictions have been spectacularly confirmed in experiments so far, what the theorem seems to show is that any candidate theory of fundamental physics must exhibit the same serious tension with special relativity that quantum theory seems to exhibit.

The worry concerning the compatibility between quantum theory and special relativity arising from the violation of Bell's formulation of local causality is highlighted and investigated in great detail in Maudlin's seminal book-length investigation [Maudlin 2011] of the topic, which, in the end, ar-

\footnotetext{
${ }^{2}$ See [Friederich 2011], [Healey 2012], [Friederich 2013], and [Friederich 2014] for recent explorations and defences of the idea that quantum states are in some sense relative to the agents who assign them and do not straightforwardly correspond to objective features of the systems they are assigned to.

${ }^{3}$ For a gentle introduction to Bell's theorem that is aimed at philosophers see [Shimony 2004], for one that is aimed at a wider scholarly readership see [Goldstein, Norsen, Tausk, and Zanghi 2011].
} 
rives at a rather pessimistic conclusion as regards the compatibility question. The central claims of Maudlin's investigation are endorsed in a more recent Scientific American article by Albert and Galchen ([Albert and Galchen 2009]), who conclude that there is indeed a "quantum threat to special relativity". The same conclusion is reached in a lucid reconstruction of Bell's original argument by Travis Norsen, who urges physicists to "appreciate that there really is here a serious inconsistency to worry about." ([Norsen 2011] p. 293) In a similar vein, Michael Seevinck contends "that a good and fair case can be made that a basic inconsistency exists between quantum theory and relativity." ([Seevinck 2010] p. 4) As these passages show, the once common view that quantum theory and special relativity are perfectly able to "peacefully coexist" ([Shimony 1978]) is nowadays under serious pressure. As a matter of fact, it has even been abandoned by Shimony, the one who historically coined the expression "peaceful coexistence". 4

The aim of the present paper is to argue, contrary to the authors just quoted and mentioned, that, properly construed, local causality is by no means violated in quantum theory. To support this claim, I will show that Bell's criterion does not adequately spell out the intuitive idea on which it is based - at least not if one accepts the widespread view that is championed, in particular, by David Lewis (see [Lewis 1980]) that objective probability ("chance") can only be what imposes constraints on rational degrees of belief in accordance with his Principal Principle. As I will argue, if one conceives of the connection between objective probability and rational credence as constitutive of what counts as objective probability, the criterion of "no superluminal signalling", which physicists normally regard as implementing the requirements of relativistic space-time structure (for more details see Section 5) is a much better candidate for an adequate implementation of local causality than the probabilistic criterion suggested by Bell. So, we have no reason for thinking that quantum theory really violates local causality, if spelled out properly, and therefore no reason, as far as Bell's criterion is concerned, to be worried that quantum theory might not be compatible with the space-time structure of special relativity.

The structure of the remaining sections of this paper is as follows:

Section 2 reviews Bell's probabilistic implementation of the intuitive criterion of local causality and highlights their motivation. Section 3 turns to the Principal Principle and recapitulates the role of the variable denoting "admissible evidence" in its formulation. Bell's criterion of local causality is critically re-examined in Section 4 in the light of the Principal Principle. Section 5 considers whether the condition of "local commutativity", which

\footnotetext{
${ }^{4}$ See Section 7 in [Shimony 2004]. An influential argument for peaceful coexistence due to [Jarrett 1984] has been thoroughly criticised in the past few years, for example by [Maudlin 2011], pp. 85-90. Others have argued that it rests on serious misunderstandings of the points Bell really wanted to make, see [Norsen 2009], [Norsen 2011] and [Näger 2013].
} 
physicists normally take to implement the causal structure of relativistic space-time, fares better than Bell's criterion and comes to a tentatively positive conclusion. The final section, Section 6, concludes the paper by summarising the assumptions on which its claims and arguments rests.

\section{Defining local causality}

\subsection{The intuitive formulation}

Bell's intuitive characterisation of what it means for a theory to be locally causal is the starting point of this discussion. For him, a theory is intuitively locally causal if, according to it, (where, to introduce my own label, "(ILC)" stands for "intuitive local causality"):

(ILC) The direct causes (and effects) of events are near by, and even the indirect causes (and effects) are no further away than permitted by the velocity of light. ([Bell 2004] p. 239.)

The criterion (ILC) has two aspects: first, that in a locally causal theory an event and its "direct" causes and effects are spatio-temporally close to each other, which means that the transmission of causal influences must somehow be "gapless"; and, second, that in a locally causal theory the transmission of causal influences may not occur at velocities larger than the velocity of light. The two aspects are related, but conceptually distinct. Bell himself, however, as well as those who have followed and accepted his main line of thought have usually focused on whether or not superluminal causal influences can occur in quantum theory and ignored the question of whether "gapless" transmission of causal influences is possible. In this paper I will follow this custom.

Fig. 1 depicts two space-time regions 1 and 2 and the (infinitely extended) space-time region 3, from which influences travelling at velocities at or below the velocity of light can reach region 1 . Region 3 is called the backward light cone of region 1. Analogously, the region "above" region 1, which influences travelling from region 1 can reach if they travel at or below the velocity of light, is called the forward light cone of region 1. Events that are neither in the backward light cone nor in the forward light cone of region 1 are said to be space-like separated from that region 1. In particular, in Fig. 1, all events in region 2 are space-like separated from all events in region 1 .

Using this terminology, we can reformulate (ILC) by saying that a theory $T$ is locally causal if and only if, according to it, an event can only be causally influenced by events which are not space-like separated from it. Furthermore, I will follow Bell in his application of the criterion (ILC) by assuming that it tacitly rules out causes in the future light cone, i.e. that 
Figure 1: Space-time structure of special relativity

it defines a theory as locally causal theory if, according to it, the causes of events lie all in their backward, rather than forward, light cones.

The main reason why superluminal causal influences are widely deemed problematic in the context of special relativity is that they are backwards in time in some inertial frames. Bell himself motivates his insistence on the importance of local causality by highlighting this point. Alluding to the paradoxical features that one often associates with backward causation, he suggests that "[t]o avoid causal chains going backward in time in some frames of reference, we require them to go slower than light in any frame of reference." ([Bell 2004] p. 236) However, even though it is true that local causality (in the sense of (ILC)) appears to be naturally motivated from the space-time structure of special relativity in the eyes of many physicists, there is no (and can be no) knock-down proof that superluminal causal influences are impossible in a theory which respects the space-time structure of special relativity. ${ }^{5}$ Moreover, as Huw Price convincingly argues, causal influences may even be "backwards in time" in all inertial frames (i.e. propagate subluminally with an inverse "sign"), without by themselves being in any

\footnotetext{
${ }^{5}$ [Maudlin 2011], pp. 141-144, elaborates on this point in great detail. While Maudlin thinks that the quantum correlations require superluminal causation, he does not regard this as by itself raising any serious problems of incompatibility between quantum theory and special relativity. What he does regard as problematic, however, is quantum theory's violation of the probabilistic criterion of local causality discussed further below.
} 
way incompatible with special relativity. ${ }^{6}$

As these considerations make clear, the question of whether special relativity really rules out superluminal causal influences and, if so, in what sense, is an intricate one. Furthermore, taken by itself, (ILC) is much too vague to permit any clear statement as regards in which theories it holds and in which it is violated. This brings us to Bell's formally precise suggestion as to how (ILC) should be implemented probabilistically.

\subsection{Probabilistic formulations}

As emphasised by Bell, the intuitive criterion of local causality (ILC) "is not yet sufficiently sharp and clean for mathematics." ([Bell 2004] p. 239.) To decide whether some theory such as quantum theory that makes probabilistic predictions conforms to local causality, what we need is a formulation of (ILC) in terms of probabilities. Bell declares that this step should be "viewed with the utmost suspicion" since "it is precisely in cleaning up intuitive ideas for mathematics that one is likely to throw out the baby with the bathwater." ([Bell 2004] p. 239) Arguably, he is right here, as becomes manifest in thatas I shall argue - his own suggested way of making (ILC) "sharp and clean for mathematics" is not the correct one if one accepts both an intuitive probabilistic criterion of local causality that he seems to accept and the Principal Principle.

The probabilistic formulation of local causality which Bell offers is cashed out in terms of what he calls "local beables". In his own words, "the beables of the theory are those entities in it which are, at least tentatively, to be taken seriously, as corresponding to something real", and he specifies that "local beables are those which are definitely associated with particular space-time regions." ([Bell 2004] p. 234) For the purposes of the present investigation, we can think of the local beables either as the objective properties of (objects within) spatio-temporally limited ("local") space-time regions or as the corresponding events, which are constituted by (the objects in) these regions' having their objective properties. Using his concept of a local beable, Bell takes local causality to be adequately captured as follows:

A theory will be said to be locally causal if the probabilities attached to the values of local beables in a space-time region 1 are unaltered by specification of values of local beables in a spacelike separated region 2 , when what happens in the backward light cone of 1 is already sufficiently specified, for example by a full specification of local beables in a space-time region 3 [see Fig. 1]. ${ }^{7}$ ([Bell 2004] pp. 239f.)

\footnotetext{
${ }^{6}$ He even gives an enthusiastic recommendation of backward causation as the clue for resolving the foundational problems of quantum theory, see Chapters 8 and 9 of [Price 1996].

${ }^{7}$ Contrary to Fig. 1, in the figure displayed in Bell's work, the space-time region 3 is
} 
This formulation is still not phrased in mathematical language and leaves room for interpretation. The essential idea behind it, however, is clear enough: in a locally causal theory any probability of a (localised) spacetime event depends only on what happens in its backward light cone and not on what occurs at space-like distance from it (nor on what occurs in its forward light cone). Let me introduce a name for this latter (still somewhat vague and intuitive) idea and call a theory "intuitively probabilistically locally causal" (in what follows "(IPLC)") if and only if according to it:

(IPLC) The probability $\operatorname{Pr}(A)$ of an event $A$ in a spacetime region 1 depends only on what events occur in the backward light cone of region 1 .

Why should one believe that only theories that fulfil the principle (IPLC) are compatible with special relativity? Maudlin argues that in quantum theory there is a "non-local dependence of one measurement result on the distant setting", which creates a compatibility problem in that it implicitly introduces a preferred reference frame the existence of which is at odds with relativity:

The underlying problem for a relativistic theory, then, is not wave collapse per se but rather the non-local dependence of one measurement result on the distant setting. In collapse theories, that dependence is secured through collapse; in Bohm's theory it is mediated through the uncollapsed wave-function. Wherever there is such a dependence then [...] there must also be a cut-off point beyond which, e.g., the setting on the right can no longer have an effect on the left, for the distant measurement can be postponed indefinitely, or never performed at all. But that cutoff point defines a preferred frame of reference, namely, the frame in which the cut-off is simultaneous with the measurement on the left. ([Maudlin 2011] p. 196.)

It seems natural to suppose that a "non-local dependence of one measurement result on the distant setting" amounts to a violation of (IPLC), i.e. a dependence of the measurement result's probability on something that is

only a subregion of the backward light cone of region 1. Crucially, as Bell emphasises, it is one which completely shields region 1 from the overlap of the backward light cones of regions 1 and 2 (as the full backward light cone of region 1 trivially does). For the purposes of this paper I identify region 3 with the full backward light cone of region 1 . The main advantages of this choice are, first, that it avoids any potential difficulties arising from hypothetical non-Markovian local theories that might incorrectly be diagnosed as not locally causal even though one sees that they are locally causal if one chooses a sufficiently large region 3 ([Goldstein, Norsen, Tausk, and Zanghi 2011], Section "Bell's definiton of locality") and, second, that it facilitates our discussion in the following sections. For a potential disadvantage see ([Goldstein, Norsen, Tausk, and Zanghi 2011], fn. 34). 
not in the backward light cone of where it occurs. For the sake of the argument, let us follow Maudlin and assume that relativity is indeed incompatible any such "non-local dependence" and that (IPLC) (or a time-symmetric cousin, if we allow backward influences) must hold in a theory that is to be compatible with special relativistic space-time structure. Does it follow that quantum theory is incompatible with relativity theory? Evidently, the question comes down to whether quantum theory violates (IPLC). But this question is remarkably difficult to answer because (IPLC) contains at least two ambiguities: first, it is unclear what should count as the probability $\operatorname{Pr}(A)$ of the event $A$; second, it is unclear what should count as the events on which $\operatorname{Pr}(A)$ depends. Nevertheless, (IPLC) seems to capture very well the intuitive content of local causality and the intuitive motivation of Bell's own formulation.

Bell himself disambiguates the involved notions by requiring that in a locally causal theory the probability $\operatorname{Pr}(A)$ of an event $A$ in region 1 , given a complete specification $E$ of what happens in the backward light cone of region 1 - in short, the probability $\operatorname{Pr}(A \mid E)$ - , be "unaltered" by specifying what happens in an arbitrary space-like separated region 2. In other words, for a probabilistic theory $T$ to be locally causal, if an event $A$ occurs in a space-time region 1 , an event $B$ in a space-like separated region 2 , and the variable $E$ specifies completely all that happens in the backward light cone of region 1 , local causality means:

$(\mathrm{BPLC}) \quad \operatorname{Pr}(A \mid E)=\operatorname{Pr}(A \mid E, B)$,

where "BPLC" stands for "Bellian probabilistic local causality". 8

The applicability of the criterion (BPLC) to quantum theory itself is not completely straightforward. The main problem in applying it is that there is no unequivocal candidate complete description $E$ of the backward light cone of the region 1 in the formal apparatus of quantum theory. The principal linguistic resources that one assigns to quantum systems (or collections of quantum systems) are quantum states $\psi$ and, as mentioned in Section 1, there is considerable dispute as to whether quantum states are descriptive at all. This dispute translates into controversy as to whether any $\psi$ can possibly give a complete descriptive account of what occurs in the backward light cone of region 1 . Unsurprisingly, based on a reading of quantum states as non-descriptive, the very applicability of (BPLC) to quantum theory has

\footnotetext{
${ }^{8}$ The present formulation of (BPLC) ignores variables $a$ and $b$ for the measurement settings in the regions 1 and 2. The full formulation reads $\operatorname{Pr}_{a}(A \mid E)=\operatorname{Pr}_{a, b}(A \mid E, B)$ ([Seevinck and Uffink 2011], Eq. (21)). I try to keep the present discussion as simple as possible by ignoring the measurement settings in my notation for the sake of simplicity. The role of the measurement settings can no longer be ignored when discussing local commutativity, as will be done in Section 5, see Eq. (5). For a useful and detailed investigation of criteria related to (BPLC), which focuses on very different aspects than the present discussion, see [Butterfield 2007].
} 
been doubted. ${ }^{9}$

If we assume, controversially, that quantum states are descriptive and that there is some quantum state $\psi$ that entails a complete description of what occurs in the backward light cone of region 1, it is plausible that (BPLC) may fail to hold for suitable $\psi$. In particular, it may fail to hold if one chooses a joint preparation method for quantum systems in region 1 and 2 such that an entangled quantum state $\psi_{E P R B}$ (where, for historical reasons, the index "EPRB" stands for "Einstein-Podolsky-Rosen" and "Bohm") must be assigned to the combined system, which predicts perfectly anti-correlated measurement results in the regions 1 and 2 if the same quantity is measured in both. For example, one can prepare a pair of electrons, one in region 1 , the other in region 2 , such that the state $\psi_{E P R B}$ to be assigned to the two ascribes probability 1 to the outcomes being either $+1 / 2$ in region 1 and $-1 / 2$ in region 2 or, conversely, $-1 / 2$ in region 1 and $+1 / 2$ in region 2 if, say, spin in $z$-direction is measured in both.

Plugging these observations in the formula (BPLC), if we denote the event "measurement of spin in $z$-direction in region 1 yields $1 / 2$ " by " $A$ " and the event "measurement of spin in $z$-direction in region 2 yields $1 / 2$ " by " $B$ ", we obtain

$$
\operatorname{Pr}\left(A \mid \psi_{E P R B}\right)=1 / 2
$$

and

$$
\operatorname{Pr}\left(A \mid \psi_{E P R B}, B\right)=0
$$

which, on the assumption that $\psi_{E P R B}$ contains a complete description of what occurs in the backward light cone of region 1 , directly contradicts (BPLC).

Based on Bell's theorem, a strong case can be made that (BPLC) is to be regarded as violated in quantum theory even if quantum states such as $\psi_{E P R B}$ are not descriptive: according to Bell's theorem, any theory must violate (BPLC) if it has the conceptual resources to give a complete description of what occurs in the backward light cone of region 1 and reproduces all the quantum theoretical predictions on correlations between outcomes. In fact, according to Bell's theorem, any such theory violates an even weaker criterion than (BPLC), where the variable $E$ describes not only what occurs

\footnotetext{
${ }^{9}$ Healey suggests this perspective on local causality: according to his preferred reading "quantum theory cannot fail to be a locally causal theory - not because it satisfies Local Causality but because that condition is simply inapplicable to quantum theory." ([Healey 2014], p. 6) Healey adds that not much hinges on whether one regards Bell's criterion as applicable to quantum theory or not: "[I]n the end it is unimportant whether one understands the condition of Local Causality to be violated by quantum theory (including relativistic quantum field theory) or simply inapplicable to that theory. [...] [O]n neither understanding does quantum theory conflict with the intuitive principle on which Bell based that condition." ([Healey 2014], p. 6) The conclusion of the present paper is in marked agreement with this last point, even though Healey reaches it along very different lines than the present paper.
} 
in the backward light cone of region 1 but everything that occurs to the "backward" side of a space-like hypersurface that intersects the backward light cone arbitrarily close to region $1 .{ }^{10}$ Since the quantum predictions on correlations between outcomes are extremely well-confirmed in experiments, it seems natural to conclude that (BPLC) is indeed violated in nature. So, if (BPLC) is the appropriate formal implementation of (IPLC), as claimed by Bell and various others, it follows that (IPLC) is indeed violated in nature as well. And if Maudlin (interpreted as above) is right, this spells trouble for special relativity.

However, as I shall argue in what follows, if we take the intuitive content of local causality to be captured by (IPLC), (BPLC) is in fact not the appropriate implementation of (IPLC) - at least if we accept the essential idea behind David Lewis' Principal Principle: that objective probability can only be what imposes constraints on rational credences.

The strategy that underlies my argument for this claim, to be presented in the following sections, is to consider what one should take to be the (unconditional) probability $\operatorname{Pr}(A)$ to insert in the intuitive criterion (IPLC), and to do so by starting from the rational credences of competent users of quantum theory. For while it is not at all straightforward what to count as the (unconditional) probability $\operatorname{Pr}(A)$, it is relatively uncontroversial what the best case rational credences are for competent users of quantum theory. As I shall argue, the best case rational credences of such users depend only on evidence concerning what occurs in their backward light cone, so the objective probabilities as well, given the Principal Principle, depend only on what occurs in the backward light cone. Accordingly, the intuitive criterion (IPLC) is fulfilled and quantum theory is, properly speaking, locally causal.

This argument obviously needs careful unpacking. In what follows, I review the essentials of Lewis's "subjectivist guide" ([Lewis 1980]) to objective probability, of which the Principal Principle is a core ingredient.

\section{The Principal Principle and admissible evidence}

To start with, I assume that the probabilities dealt with in quantum theory are in some sense objective. And indeed they are manifestly objective inasmuch as their ascriptions are governed by objective standards of correctness that are (at implicitly) acknowledged by those who apply the theory in practice. But, we may ask, in virtue of which of their features are these objective quantities objective probabilities for certain events to occur? It is precisely in order to answer questions of this type that David Lewis recommends the link between objective probability ("chance", as he calls it) and rational degree of belief ("rational credence") which he calls the Principal

\footnotetext{
${ }^{10}$ I would like to thank an anonymous referee for suggesting to highlight this point.
} 
Principle as a guide to the identification of quantities which deserve to be called "probabilities" (or "chances", in his terminology). As he urges:

Don't call any alleged feature of reality 'chance' unless you've already shown that you have something, knowledge of which could constrain rational credence. ([Lewis 1994], p. 484)

The Principal Principle itself states that, for any coherent prior credence function $\operatorname{cr}()^{11}$ and any arbitrary proposition $A$ concerning some chancy matter of fact ${ }^{12}$, the quantity $x$ deserves to be called the "objective probability" of $A$ being true $-\operatorname{Pr}(A)$, for short - only if (where "(PP)" stands for "Principal Principle")

$$
(P P) \quad \operatorname{cr}(A \mid \operatorname{Pr}(A)=x, E)=x,
$$

where the variable $E$ denotes what Lewis calls "admissible evidence"13 (on which more in a moment). Conversely, if $x$ is the objective probability of $A$ being true, as one may happen to know in some specific case on independent grounds, then, according to the Principal Principle, the degree of belief one should have as to whether $A$ will be $x$.

Evidence concerning $A$ which allows to make more informed judgements as to whether $A$ than possible on the basis of evidence concerning $A$ 's chance is called "inadmissible". As the term suggests, such evidence is not part of the "admissible" evidence $E$ in (PP). The simplest example of inadmissible evidence is direct evidence that $A$ has indeed occurred (or will occur). For example, if you happen to take part in the event of a fair coin toss and therefore have direct evidence as regards its outcome, your rational credences as regards the outcomes HEADS and TAILS are 0 and 1, whereas the objective probability for the possible outcomes may still be taken to be $1 / 2$. Specifying what evidence is admissible is the real challenge when putting the Principal Principle to philosophical work, because, depending on what evidence one regards as admissible, one arrives at differing judgements as to what degrees of belief are rational or (if one is not in doubt about what degrees of belief are rational) as to what the objective probabilities are. What is clear, however, is that evidence concerning the objective probability $\operatorname{Pr}(A)$ of $A$ must always be admissible for an agent for whom the rational credence, dictated by the Principal Principle, equals $\operatorname{Pr}(A)$. Evidence can be inadmissible only if having it makes it potentially rational not to align one's credence about $A$ with $A$ 's objective probability. It follows that evidence as regards $A$ 's objective probability itself is always admissible.

\footnotetext{
${ }^{11}$ See [Lewis 1980] pp. 87-89 for details.

${ }^{12}$ In what follows I will use the letter " $A$ " to denote both the proposition concerning some chancy fact as well as the "chancy" event itself. So, the expression " $\operatorname{Pr}(A)$ " can either be read as "the chance of (the proposition) $A$ being true" or as "the chance of (the event) $A$ to occur".

${ }^{13}$ Ibid. pp. $92-96$.
} 
Agents at different spatio-temporal locations have in general access to different bits of evidence, so their rational credences with respect to one and the same proposition $A$ may differ. Lewis himself takes the agent-relativity of rational credences (which translates into a relativisation of objective probabilities) into account by relativising total amounts of admissible evidence with respect to time. Given the Principal Principle, assuming total amounts of admissible evidence to be relative to the time parameter $t$ is equivalent to treating objective probabilities as time-dependent. Lewis formulates the Principal Principle in terms of a theory $T$ from which chances are derived and a history $H_{t}$ of the world up to the time $t$ as

$$
(T P P) \quad \operatorname{cr}\left(A \mid H_{t}, T\right)=\operatorname{Pr}_{t}(A)^{14}
$$

(where in my terminology "(TPP)" stands for "time-relativised Principal Principle"). According to how (TPP) is to be read following Lewis, $H_{t}$ is a complete description of the world up to the time $t$ where $\operatorname{Pr}$ is evaluated. So, an assumption which enters here is that evidence about one's past is always admissible, whereas evidence about one's future is generally not. The problem with this formulation of the Principal Principle, for our present purposes, is that in the context of special relativistic space-time there is no preferred relation of simultaneity among events and hence no preferred timeparameter that could play the role of the variable $t$ in (TPP). A fortiori, there is no preferred way to carve up space-time into past and future, and the formulation (TPP) of the Principal Principle, with its relativisation of objective probabilities with respect to time, cannot be used as it stands.

At first glance, the most natural reaction to this difficulty is to relativise chances with respect to space-like hyperplanes, i.e. planes of constant time $t$ in a specific inertial frame. Wayne Myrvold, for example, proposes this move as a promising step to make sense of "relativistic quantum becoming" (the title of [Myrvold 2003]). And indeed, as he correctly observes, "it is not nonsensical to conditionalise on events not in the past" ([Myrvold 2003] p. 492) (or, in the relativistic context, outside the backward light cone), which means that quantities $\operatorname{Pr}_{E_{h}}(A)$ obtained by conditionalising the probability function $P r$ with respect to everything that occurs on one side of the space-like hyperplane $h$ may be well-defined. This leaves open the question, however, whether the resulting quantities deserve to be regarded as objective probabilities in the sense of the Principal Principle. As it turns out, the quantities $\operatorname{Pr}_{E_{h}}(A)$ are arguably not the probabilities we are after, because they do not specify the rational credences for agents such as us (as they would have to, if they were the searched for probabilities) for the following reasons:

Hyperplanes are indefinitely stretched out in space-time; so, if we identify the rational credence of some agent on earth with $\operatorname{Pr}_{E_{h}}(A)$, there is no

\footnotetext{
${ }^{14}$ See [Lewis 1980] p. 97 as "the Principal Principle reformulated".
} 
reason as to why we should not do the same for the rational credence of some (hypothetical) agent located myriads of light years away. However, the rational credences of the agent here on earth and those of the agent myriads of light years away, for example with respect to matters of fact on earth, are clearly not the same, even if both their space-time curves happen to intersect the same hyperplane $h$ at some point: both have access to very different bits of information about what happens in the universe, and this makes it overwhelmingly plausibly that their rational degrees of belief are in general different.

Unlike agents who are located along the same space-like hyperplane, agents who are located in the same (finite and not overly large) space-time region may at least in principle share all the information they have as regards what events have occurred (or will occur) across space-time. So, if we accept some modest and natural degree of idealisation, it makes sense to assume that rational credences - and thereby, by the Principal Principle, the objective probabilities themselves - are relative to those (finite and not overly large) space-time regions. ${ }^{15}$

Interestingly, space-time regions which fit the bill of being finite and not overly large have already played a role in the present investigation, namely, in the above discussion of local causality, where chancy events $A$ and $B$ were attributed to the space-like separated space-time regions 1 and 2 . The idea which naturally comes to mind at this step is that regions of this type are precisely what objective probabilities should be relativised to in the context of special relativistic space-time. For the sake of brevity, I neglect the many further questions which arise at this point, e.g. concerning the exact size and form of these regions and concerning whether they might be chosen pointlike. Assuming that these questions can be settled in a satisfying manner, we can introduce a variable $y$ to range over the chosen space-time regions and arrive at the following reformulation of the Principal Principle (where "(RPP)" stands for "region-relativised Principal Principle"):

$$
(R P P) \quad \operatorname{cr}\left(A \mid E_{y}, T\right)=\operatorname{Pr}_{y}(A) .
$$

Here again " $T$ " denotes the theory which we use to compute the probabilities and " $E_{y}$ " now denotes the total amount of admissible evidence relative to the space-time region $y$.

Before concluding this section, it is worth adding that, as demonstrated by Ned Hall, one can formulate the Principal Principle without recourse

\footnotetext{
${ }^{15}$ As persuasively argued by [Cusbert 2013], there are strong reasons for conceiving of chances as relative to causal histories rather than times. It seems natural to suppose that in typical "chance theories" in the special relativistic space-time framework causal histories will approximately coincide for all points in a finite and not overly large space-time region, whereas they will differ for distant points on the same space-like hypersurface. So, if Cusbert is right, this provides us with further reasons for expecting objective probabilities to be relative to "local" space-time regions rather than space-like hypersurfaces.
} 
to the notion of admissible evidence. Hall achieves this by providing an explicit definition of admissibility that allows to eliminate the notion from the Principal Principle. Hall's definition (Eq. (3.13) in [Hall 2004]) states that evidence $F$ is admissible with respect to the credence function $\mathrm{cr}$ if and only if $c r$ assigns probability 1 to " $c h_{t}(A)=c h_{t}(A \mid F)$ ", where $c h_{t}(A)$ is the same as $\operatorname{Pr}_{t}(A)$ in (TPP). If we focus on a single chance theory $T$ and assume that the agents we consider are completely certain that $T$ is correct, their credence function $c r$ will assign probability 1 to " $c h_{t}(A)=$ $c h_{t}(A \mid F)$ " just in case this follows from $T$. Since in this paper we focus on a single chance theory (quantum theory) and assume that all (hypothetical) spatio-temporally situated agents are certain that this theory is correct, this condition is fulfilled, and Hall's criterion of admissibility for $F$ reduces to "cht $(A)=c h_{t}(A \mid F)$ " (not including any mention of the credence function cr). ${ }^{16}$

One way of paraphrasing Hall's criterion of admissibility is by saying that the chances are so that arbitrary bits of admissible evidence $F$, when conditionalised over, are irrelevant for (i.e. without impact on) the chances. This may appear to provide us with a straightforward way of defining chances and admissible evidence such that the criterion is fulfilled by construction: first, start with some amount of evidence $E^{1}$ that is uncontroversially admissible and consider $\operatorname{Pr}_{y}\left(A \mid E^{1}\right)$. Then consider some further amount of evidence $F^{1}$ that is not included in $E^{1}$ and add it to the provisionally defined admissible evidence $E^{1}$ (which will thus be replaced by $\left.E^{2}=E^{1}, F^{1}\right)$ if and only if $F^{1}$ has a non-trivial impact on the chances in that $\operatorname{Pr}_{y}\left(A \mid E^{1}, F^{1}\right) \neq \operatorname{Pr}_{y}\left(A \mid E^{1}\right)$. Repeat this process of adding more and more admissible evidence until you arrive at what will turn out to be the definitive total amount of admissible evidence $E^{N}$, for which there is now no further evidence $F^{N}$ such that $\operatorname{Pr}_{y}\left(A \mid E^{N}, F^{N}\right) \neq \operatorname{Pr}_{y}\left(A \mid E^{N}\right)$. For all those $F^{i}$ that have been included in the construction of $E^{N}$ in this procedure, the criterion " $\operatorname{Pr}_{y}\left(A \mid E^{N}, F^{i}\right)=\operatorname{Pr}_{y}\left(A \mid E^{N}\right)$ " now holds by construction. By defining the unconditional chance as $\operatorname{Pr}_{y}(A):=\operatorname{Pr}_{y}\left(A \mid E^{N}\right)$ one has made it trivially true that $\operatorname{Pr}_{y}(A)=\operatorname{Pr}_{y}(A \mid F)$ for all bits of admissible evidence $F \subset E^{N}$, just as Hall's criterion of admissibility requires.

Interestingly, if we apply this strategy to the specification of unconditional probabilities and complete amounts of admissible evidence in quantum theory, we obtain the result that $B$ in Fig. 1 is admissible evidence relative to the region 1 . This result follows directly from the fact that

\footnotetext{
${ }^{16}$ This allows me to ignore a further complication that Hall criterion successfully addresses, namely, the difficulty that, according to Lewis' criterion (TPP), a pattern of events which according to the chance theory $T$ can occur with nonzero probability may undermine $T$ as the correct chance theory of the world. The problem is solved by replacing the original principle (TPP) with the modified "New Principle" that reduces to the original one if there is only one candidate chance theory T. ([Lewis 1994], p. 487 and [Hall 1994], p. 511)
} 
$\operatorname{Pr}_{1}(A \mid E) \neq \operatorname{Pr}_{1}(A \mid E, B)$, i.e. from the fact that (BPLC) is violated in quantum theory. The event $B$ must be included in the admissible evidence relative to the region 1 , one might argue, because $B$ is non-trivially relevant for the rational credences of whoever happens to be situated in the region 1.

Contrary to this line of thought, as I shall argue below, evidence about $B$ is in fact inadmissible with respect to the region 1 . However, at the present stage we only need to note that the strategy just sketched to determine the unconditional probability $\operatorname{Pr}_{y}(A)$ cannot possibly be adequate. The main problem with it is that, according it, for arbitrary $A$ the value of $\operatorname{Pr}_{y}(A)$ must be 0 or 1 . To see this, assume, for reductio, that there is some $A$ for which $0<\operatorname{Pr}_{y}(A)=\operatorname{Pr}_{y}\left(A \mid E^{N}\right)<1$. Assuming that $A$ does occur, one has $\operatorname{Pr}_{y}\left(A \mid E^{N}\right) \neq \operatorname{Pr}_{y}\left(A \mid E^{N}, A\right)=1$, contrary to how $E^{N}$ has been constructed. Analogously, assuming that $A$ does not occur, one has $\operatorname{Pr}_{y}\left(A \mid E^{N}\right) \neq \operatorname{Pr}_{y}\left(A \mid E^{N}, \neg A\right)=0$, contrary to how $E^{N}$ has been constructed. Since at least for some $A$ the probability $\operatorname{Pr}_{y}(A)$ should certainly be neither 0 nor 1 , it follows that the admissible evidence cannot be determined in the manner just outlined. In particular, the mere fact that, as discussed in the end of Section 2, (BPLC) is violated in quantum theory in that $\operatorname{Pr}_{1}(A \mid E) \neq \operatorname{Pr}_{1}(A \mid E, B)$ does not establish that $B$ is admissible with respect to region $1 .{ }^{17}$

To conclude, what we need is an independent way of specifying $\operatorname{Pr}_{y}(A)$ or (what amounts to the same) an independent way of specifying the complete admissible evidence $E_{y}$ so that - in conformity with Hall's criterion$\operatorname{Pr}_{y}(A)=\operatorname{Pr}_{y}\left(A \mid E_{y}\right)$. The strategy used in what follows is to obtain $E_{y}$ in quantum theory by considering the rational credences of competent users of quantum theory in those cases where they have all the possibly relevant evidence that they can have if quantum theory is correct and to conclude from there what the total amounts of admissible evidence are. To achieve

\footnotetext{
${ }^{17}$ Hall's account of admissible evidence does in fact have subtly different implications from Lewis', but these can be neglected for our present purposes: Lewisian chances come apart from Hallian chances if agents have evidence that allows them to have more informed rational credences than those obtained on grounds of the chance theory that they use. To see what this means in practice, assume that, according to the chance theory $T$ that is used, only evidence about the past is admissible. Then, according to Lewis, if the agent acquires evidence about the future due to reliable oracles and the like (referred to as "crystal balls" by [Hall 1994], p. 508), this evidence is inadmissible. Nevertheless, in order for her credences to be rational, she may need to take such evidence into account, so rational credences will no longer coincide with the chances. According to Hall, in contrast, any evidence that an agent has automatically counts as admissible, even if obtained by the help of crystal balls. As a consequence, on the Hallian account the rational credences coincide in all circumstances with the chances, but the chances are not in all cases those derived from the chance theory $T$, according to which only evidence about the past is admissible. For the sake of simplicity, I assume for our present purposes that there are no "crystal balls" around or, to consider the more general case, no exemptions from the chance theory $T$ - quantum theory - that our agents use.
} 
this aim, we will first need to formulate the intuitive probabilistic criterion of local causality (IPLC) in the language of the Principal Principle.

\section{Local causality in the light of the Principal Prin- ciple}

\subsection{Probabilistic local causality and (in)admissible evidence}

Using the conceptual apparatus developed in the previous section it is easy to express the intuitive probabilistic criterion of local causality (IPLC) in the language of the Principal Principle and compare it with Bell's criterion (BPLC). The criterion (IPLC), recall, says that in a locally causal theory the probability of an event $A$ in a space-time region 1 depends only on what occurs in the backward light cone of that region.

The first question we have to address is which probability $\operatorname{Pr}_{y}(A)$ (i.e. $\operatorname{Pr}_{y}(A)$ for which value of the variable $y$ ) we should consider as the probability of $A$ in the sense of (IPLC). It seems to me that the only non-arbitrary choice is the probability $\operatorname{Pr}_{1}(A)$, i.e. the probability relative to the spacetime region 1 itself, where $A$ potentially occurs. By the Principal Principle, this is equivalent to the rational credence concerning $A$ for an agent who is associated with the space-time region 1; most plausibly by being located at, or at least very close, to that region $1 .{ }^{18}$

In the terminology of the Principal Principle, the probability $\operatorname{Pr}_{1}(A)$ of $A$ relative to the space-time region 1 is a function of the total admissible evidence $E_{1}$ relative to the region 1 . Now consider $E_{1}$ represented as the conjunction of the total admissible evidence $E_{1-}$ about what happens inside the backward light cone of 1 and the total admissible evidence $E_{1,0}$ about what happens outside its backward light cone: $E_{1}=E_{1-}, E_{1,0}$. The intuitive principle of probabilistic local causality (IPLC) now requires that, for $T$ to be locally causal, $\operatorname{Pr}_{1}(A)$ according to the theory $T$ may depend only on what happens in the backward light cone of region 1, i.e. only on $E_{1-}$. In other words, the principle (IPLC) mandates that $\operatorname{Pr}_{1}(A)$ (which is defined as $\left.\operatorname{Pr}_{1}\left(A \mid E_{1}\right)\right)$ must be equal to $\operatorname{Pr}_{1}\left(A \mid E_{1-}\right)$ :

$$
\operatorname{Pr}_{1}(A)=\operatorname{Pr}_{1}\left(A \mid E_{1-}\right) .
$$

Arguably, Eq. (3) is the appropriate formal expression of what it takes for a theory to be locally causal by the standards of (IPLC) in the light of the Principal Principle. Eq. (3) is formulated in terms of objective probabilities

\footnotetext{
${ }^{18}$ In what follows, I assume that the admissible evidence for the agent in region 1 does not include the event $A$ itself, for otherwise the associated probability $\operatorname{Pr}_{1}(A)$ would be trivial (i.e. 0 or 1 ). The problem we encounter here is essentially that of specifying the probability of an event with respect to the very moment where it occurs (or fails to occur). Since this problem is in no way specific to the present investigation, I feel free to ignore it here.
} 
no less than Bell's criterion (BPLC). We can thus compare the two and see whether they yield the same verdicts as to which theories are locally causal and which not.

Potential differences between Eq. (3) and (BPLC) arise from the fact that the latter does not pay attention to admissibility. Let us assume (as an inessential simplification) that all evidence about what occurs in the backward light cone is admissible and denote by " $I_{1}$ " the complete inadmissible evidence about what happens at space-like separation from region 1 (if such there be). Furthermore, let us assume that there is no admissible evidence about what occurs in the forward light cone. (BPLC) states that $\operatorname{Pr}(A \mid E, B)=\operatorname{Pr}(A \mid E)$ for arbitrary events $B$ at space-like separation from the region 1 , whether $B$ is admissible or not. So, given these assumptions, we can rephrase (BPLC) as

$$
\operatorname{Pr}_{1}\left(A \mid E_{1}, I_{1}\right)=\operatorname{Pr}_{1}\left(A \mid E_{1-}\right) .
$$

In words: the conditional probability of $A$, given everything that occurs either in the backward light cone of the region 1 or at space-like distance from it (both what is admissible and what is inadmissible), is the same as the conditional probability of $A$, given only what occurs in the backward light cone of region 1 (which, as assumed, is all admissible).

Are the criteria Eq. (3) and Eq. (4) equivalent? Remember that $\operatorname{Pr}_{1}(A)$ on the left hand side of Eq. (3) is the same as $\operatorname{Pr}_{1}\left(A \mid E_{1}\right)$. Clearly, the two criteria are not equivalent if there is nontrivial inadmissible evidence $I_{1}$ about what occurs at space-like distance from region 1 . If such evidence exists, their verdicts about the theory $T$ at issue may differ. In particular, if for some theory $T$ the complete admissible evidence $E_{1}$ with respect to region 1 can be derived from evidence about the backward light cone-i.e. if $E_{1}$ and $E_{1-}$ are equivalent from the point of view of the theory $T$-, then Eq. (3) holds trivially for $T$, whereas Eq. (4) and, thus, Bell's criterion (BPLC), may fail to hold. In Section 5 I argue that this is plausibly true about quantum theory.

To find out whether Eq. (3) holds in quantum theory, we need to determine the total admissible evidence $E_{1}$ relative to the region 1 . The next subsection mentions an important principle that allows to narrow it down.

\subsection{Admissible evidence and "ought implies can"}

The Principal Principle is a normative principle in that it dictates the credences that an agent rationally ought to have, given her knowledge of objective probabilities. As already remarked, what an agent's rational credences are depends on what evidence she can dispose of (by conditionalising with respect to it) to arrive at them. Arguably, one of the best-known maxims of practical philosophy has an impact on the relation between rational credence and available evidence at this point, namely, the maxim "ought 
implies can". An instance of this principle that is straightforwardly relevant to our present problem is that an agent's rational credences - those that she rationally ought to have-depend on what evidence she can possibly have, given how she is spatio-temporally situated.

Let me illustrate this point by considering an agent who uses some probabilistic theory $T$ to determine her rational credences and by assuming that, according to $T$, she cannot possibly have any evidence as to whether some chancy event $B$ does occur (has occurred) or not due to where she is situated. Then, in accordance with "ought implies can", she may not be held responsible for not having any evidence that $B$ does in fact occur (or has occurred). A fortiori, she may not be held responsible for not taking such evidence into account when forming her credences, even if differently situated agents have that evidence. Consequently, evidence that $B$ must be inadmissible relative to her position.

To further highlight this point, let us assume that our agent has a "hunch" that $B$ occurs and that, as a matter of fact, $B$ does indeed occur. Nevertheless, it remains irrational for our agents to trust her hunch and to form her credences by conditionalising with respect to $B$, for (by assumption) her rational trust is in $T$, which tells her that she cannot have any evidence as to whether $B$. This remains so independently of whether forming novel credences based on the assumption that $B$ does occur (or has occurred) enhances her predictive and pragmatic success. If it does, she is well described as a "lucky fool" by irrationally following her hunch that $B$ occurs (has occurred).

To conclude, for evidence to be admissible with respect to some $y$ according to the theory $T$, agents located at $y$ must be able to access $B$ if $T$ is correct. Thus, returning to quantum theory and the criterion of local causality Eq. (3), what we have to ask is whether all the evidence that an agent who is located at $y$ has access to is the evidence $E_{-1}$ about her backward light cone (plus what she can derive from $E_{-1}$, using $T$ ).

As I argue in what follows, quantum theory seems to meet this criterion in the light of the "no superluminal signalling theorem". That is, by the assumptions made, quantum theory seems to be perfectly locally causal in the sense of (IPLC) as spelled out in the form of Eq. (3).

\section{Quantum theory, local causality, and the Prin- cipal Principle}

The assumption which physicists normally take to implement the requirements of relativistic space-time structure in a relativistic quantum theory is that the mathematical objects ("operators") which represent the dynamical variables ("observables") of physical systems commute if they are associated with space-like separated space-time regions or points. In more formal 
terms, if $x$ and $y$ are space-like separated space-time points and $A(x)$ and $B(y)$ are operators representing dynamical variables associated with these points, one puts forward the assumption: ${ }^{19}$

$$
[A(x), B(y)]=A(x) B(y)-B(y) A(x)=0 .
$$

The physical significance of (5), according to the shared understanding of competent users of quantum theory, is that an agent's choice to measure the observable $A(x)$ at (or close to) the space-time point $x$ is statistically irrelevant for the outcomes of arbitrary further measurements of observables $B(y)$ at (or close to) the space-like separated space-time point $y$. In particular, it is impossible for an agent located at $x$ to send any signal to an agent located at $y$ by deciding to measure $A(x)$ rather than some other $A^{\prime}(x)$. This statement can be given a mathematically precise form and, as such, is known as the "no-signalling theorem". ${ }^{20}$ Physicists widely regard this principle of no- (superluminal) signalling as the correct formal implementation of the causal structure of relativistic space-time, which is why the assumption Eq. (5) is often referred to as "relativistic causality", "microcausality", or just "causality". ([Haag 1993] p. 57) We may now ask how Eq. (5) - and, with it, the impossibility of superluminal signalling - fares as a way of implementing local causality in the light of the previous discussion based on the the Principal Principle. Does it make sure that Eq. (3) holds in quantum theory?

To begin with, an obvious difference between Eq. (5) on the one hand and (IPLC) as well as Eqs. (3) and (4) on the other is that the first is timesymmetric, whereas the latter are not. It follows that Eq. (5) can at best be the formal implementation of a time-symmetric cousin of (IPLC) which refers to the total (backward and forward) light cone of the space-time region 1 and not only to its backward light cone. However, setting aside the thorny issue of backward in time dependences of probabilities, we can still focus on whether Eq. (5) ensures that Eq. (3) holds inasmuch as the latter rules out that (unconditional) probabilities depend on what occurs at space-like separation.

That Eq. (5) holds and superluminal signalling is impossible is surely a necessary condition for Eq. (3): in a theory $T$ which allows superluminal signalling, evidence as regards what occurs at space-like separation is in principle accessible (via superluminal signals), so rational agents have no general excuse as to why they may neglect it when forming their credences. Consequently, evidence concerning space-like separated events will typically be admissible in theories that permit superluminal signalling and

\footnotetext{
${ }^{19}$ See [Gell-Mann, Goldberger and Thirring 1954] for a historically important reference. See [Haag 1993] (p. 57 and p. 107) for a version in the language of the mathematically rigorous algebraic approach to quantum theories.

${ }^{20}$ See [Eberhard 1978, Ghirardi, Rimini, and Weber 1980] for versions and proofs.
} 
will, hence, have a non-trivial impact on the objective probabilities. Thus, Eq. (3) cannot be expected to hold in a theory that allows superluminal signalling.

In a theory $T$, in contrast, in which superluminal signalling is impossible, any evidence as regards what happens at space-like separation from an agent is inaccessible for her unless she can derive it, using $T$, from what occurs in her own backward light cone (or total light cone, if we allow backwards in time dependences of probabilities). ${ }^{21}$ If this is true, then, according to the considerations on the relevance of "ought implies can" in Section 4.2, agents' rational credences in such theories are not based on any evidence as to what occurs at space-like distance. Quantum theoretical practice seems to strongly support this view: agents using quantum theory are in fact not held responsible for (not) taking into account what occurs at space-like separation from them if they cannot derive it, using quantum theory itself, from what occurs in their backward light cone. And the reason why they are not held responsible seems to be that due to Eq. (5) and its physical implications such evidence is considered inaccessible to them. If this diagnosis is correct, the complete admissible evidence in quantum theory reduces to evidence about what occurs in the backward light cone-i.e. $E_{1}, T$ is equivalent to $E_{1-}, T$-, which implies that Eq. (3) holds in quantum theory. And this in turn, finally, means that quantum theory is locally causal in the sense of (IPLC) when that principle is spelled out in the light of the Principal Principle.

Establishing that quantum theory does not violate local causality in this sense is of course not the same as establishing that there is no problem of compatibility between quantum theory and special relativity. Interpretations of quantum theory may invoke such notions as absolute simultaneity and a preferred reference frame, which are difficult or impossible to reconcile with a fully Lorentz invariant account. This concerns, in particular, (versions of) pilot wave theory and GRW-theory. Recall, however, that for Maudlin (see the quotation in Section 2.2), there exists an inevitable conflict between quantum and relativity theory due to what he calls "the non-local dependence of one measurement result on the distant setting" ([Maudlin 2011] p. 196) in quantum theory. Contrary to this view, the present considerations allow for a more optimistic perspective: inasmuch as the incompatibility worry rests on the belief that quantum theory violates local causality in that quantum probabilities are influenced by what occurs at space-like distance, it may well be laid to rest. ${ }^{22}$

\footnotetext{
${ }^{21}$ She may also derive it from what occurs now where she is together with evidence about her backward light cone. Evidence about what occurs in the backward light cone may include, for example, memories of agreements that were made as to which obervables are to be measured in the future at space-like separation from her.

${ }^{22}$ There are further potentially interesting ramifications of the considerations offered here. For example, if we interpret the present argument to the end that (IPLC) is not
} 


\section{Concluding remarks}

The present case for the claim that quantum theory is, properly speaking, locally causal rests on a number of assumptions that critics may challenge: first, that the intuitive probabilistic principle (IPLC) according to which probabilities in a locally causal theory depend only on what occurs in the backward light cone adequately captures the idea of local causality; second, that objective probability can only be what imposes constraints on the rational credences of (actual or hypothetical) agents, as encoded in the Principal Principle; third, that in the special relativistic space-time framework objective probabilities are to be conceived of as relative to "local" space-time regions of the sort considered here; fourth, that an agent's rational credences never depend on matters that he or she cannot have any evidence of (here is where "ought implies can" comes in); and, fifth, that if superluminal signalling is impossible, agents do not have access to evidence about what occurs at space-like separation unless they can derive it, using quantum theory, from what occurs in their backward light cone (total light cone, if we allow backward influences).

Any of these assumptions may conceivably be challenged, but none of them should be dismissed lightly. To accept them all is an attractive option in that it leads to a vindication of the gut feeling (and, in fact, the mainstream view) among contemporary physicists that there is no genuine "non-locality" in quantum theory at all and that, a fortiori, the violation of Bell's criterion (BPLC) in quantum theory does not indicate any incompatibility between quantum theory and relativity. Given the empirical success of the theories of modern particle physics, which are based on combining the central principles of these two "pillars of contemporary theory" (Bell), this is surely an attractive perspective. ${ }^{23}$

violated in quantum theory as suggesting that (ILC) is not violated as well and that, hence, the quantum correlations are non-causal (which admittedly would require further argument), then, as persuasively argued by Cavalcanti (see [Cavalcanti 2010]), this may spell trouble for causal (as opposed to standard evidential) decision theory in that it recommends losing betting strategies in gambles that refer to EPRB-type setups.

${ }^{23}$ A remaining worry that one might have is how nature manages to "perform the trick" (see the abstract of [Gisin 2009]) of producing correlations that violate (BPLC) without availing herself of non-local influences that are incompatible with the spirit, if not the letter, of relativity theory. In [Friederich 2014], Chapter 10.6, I argue that this worry rests on a misguided picture of the role of time in the "coming about" of the quantum correlations. Once the block universe view is accepted as the mature perspective on the role and status of time the question of how nature might be able to produce certain correlations is no longer well posed. 


\section{Acknowledgments}

I would like to thank Andreas Bartels, Jeremy Butterfield, Michael Esfeld, Thorben Petersen, two anonymous referees, and the members of the Göttinger Philosophisches Reflektorium for useful comments on earlier versions. I am grateful to discussions with conference audiences in Hanover and Munich, where the material developed here was presented.

\section{References}

[Albert and Galchen 2009] Albert, D. Z and Galchen, R. (2009), Was Einstein wrong?: A quantum threat to special relativity, Scientific American, March 2009: 32-39.

[Bell 2004] Bell, J. S. (2004), Speakable and Unspeakable in Quantum Mechanics, 2nd edition, Cambridge: Cambridge University Press.

[Butterfield 2007] Butterfield, J. N. (2007), Stochastic Einstein locality revisited, British Journal for the Philosophy of Science 58:805-867.

[Cavalcanti 2010] Cavalcanti, E. G. (2010), Causation, decision theory, and Bell's theorem: a quantum analogue of the Newcomb problem, British Journal for the Philosophy of Science 61:359-597.

[Cusbert 2013] Cusbert, J. (2013), The Arrow of Chance, PhD thesis, submitted at The Australian National University.

[Eberhard 1978] , Eberhard, P. H. (1978), Bell's theorem and the different concepts of locality, Nuovo Cimento 46B, 392-419.

[Friederich 2011] Friederich, S. (2011), How to spell out the epistemic conception of quantum states, Studies in History and Philosophy of Modern Physics 42:149-157.

[Friederich 2013] Friederich, S. (2013), In defence of non-ontic accounts of quantum states, Studies in History and Philosophy of Modern Physics 44:77-92.

[Friederich 2014] Friederich, S. (2014), Interpreting Quantum Theory: A Therapeutic Approach, Houndmills, Basingstoke, Hampshire: Palgrave Macmillan.

[Gell-Mann, Goldberger and Thirring 1954] Gell-Mann, M., Goldberger, M. L., and Thirring, W. E. (1954), Use of causality conditions in quantum theory, Physical Review 95:1612-1627. 
[Ghirardi, Rimini, and Weber 1980] Ghirardi, G. C., Rimini A., and Weber, T. (1980), A general argument against superluminal transmission through the quantum mechanical measurement process, Lettere al Nuovo Cimento 27:293-298.

[Gisin 2009] Gisin, N. (2009), Quantum nonlocality: How does nature do it?, Science 326:1357-1358.

[Goldstein, Norsen, Tausk, and Zanghi 2011] Goldstein, S., Norsen, T., Tausk, D. V., and Zanghi, N. (2011), Bell's theorem, Scholarpedia, $6: 8378$.

[Haag 1993] Haag, R. (1993), Local Quantum Physics, corrected edition, Berlin: Springer.

[Hall 1994] Hall, N. (1994), Correcting the guide to objective chance, Mind 103:505-517.

[Hall 2004] Hall, N. (2004), Two mistakes about credence and chance, Australasian Journal of Philosophy 82:93-111.

[Healey 2012] Healey, R. A. (2012), Quantum theory: a pragmatist approach, British Journal for the Philosophy of Science 63:729-771.

[Healey 2014] Healey, R. A. (2014), Causality and chance in relativistic quantum field theories, Studies in History and Philosophy of Modern Physics, http://dx.doi.org/10.1016/j.shpsb.2014.03.002

[Jarrett 1984] Jarrett, J. (1984), On the physical significance of the locality conditions in the Bell arguments, Nous 18:569-589.

[Lewis 1980] Lewis, D. (1986 [1980]), A subjectivists's guide to objective chance, In: Philosophical Papers, Vol. II, New York: Oxford University Press, 83-132 (originally published in: Studies in Inductive Logic and Probability, Vol. II, ed. Richard C. Jeffrey, Berkeley: University of California Press).

[Lewis 1994] Lewis, D. (1994), Humean supervenience debugged, Mind 103: 473-490.

[Maudlin 2011] Maudlin, T. (2011), Quantum Theory and Relativity Theory: Metaphysical Intimations of Modern Physics, 3rd ed., Oxford: Wiley-Blackwell.

[Myrvold 2003] Myrvold, W. C. (2003), Relativistic quantum becoming, British Journal for the Philosophy of Science 54:475-500.

[Näger 2013] Näger, P. (2013), A stronger Bell argument for quantum nonlocality, http://philsci-archive.pitt.edu/9932/. 
[Norsen 2009] Norsen, T., Local causality and completeness: Bell vs. Jarrett, Foundations of Physics 39: 273-294.

[Norsen 2011] Norsen, T., John S. Bell's concept of local causality, American Journal of Physics 79:1261-1275.

[Price 1996] Price, H. (1996), Time's Arrow and Archimedes' Point: New Directions for the Physics of Time, Oxford, New York: Oxford University Press.

[Seevinck 2010] Seevinck, M. P. (2010), Can quantum theory and special relativity peacefully coexist?, available at http://arxiv.org/abs/1010.3714.

[Seevinck and Uffink 2011] Seevinck, M. P. and Uffink, J. (2011), Not throwing out the baby with the bathwater: Bell's condition of local causality mathematically 'sharp and clean', In: Explanation, Rediction and Confirmation. New Trends and Old Ones Reconsidered, D. Dieks, W.J. Gonzalez, S. Hartmann, Th. Uebel, and M. Weber (eds.) Dordrecht: Springer, 425-450.

[Shimony 1978] Shimony, A. (1978), Metaphysical problems in the foundations of quantum mechanics, International Philosophical Quarterly 18: 3-17.

[Shimony 2004] Shimony, A. (2004), Bell's theorem, The Stanford Encyclopedia of Philosophy (Winter 2012 Edition), E. N. Zalta (ed.), $<$ plato.stanford.edu/archives/win2012/entries/Bell-theorem>. 